\title{
Targeting Keloid Fibroblasts by Inhibition of Hypoxia Signaling
}

Journal of

\section{Clinical \& Investigative} Dermatology

\section{Richert-Jones J1', Mantel A' ${ }^{1}$, Ricks-Santi L ${ }^{2}$, Harvey $\mathbf{V}^{1}$} and Chan $\mathrm{J}^{1^{*}}$

${ }^{\prime}$ Hampton University Skin of Color Research Institute, USA

${ }^{2}$ Hampton University Cancer Research Center, USA

\section{*Address for Correspondence}

Joanne Chan, Scientific Director and Associate Professor, Hampton University Skin of Color Research Institute (HUSCRI) 100 William R. Harvey Way, Hampton, VA 23668, Hampton, VA 23668, USA, Tel: 757-726-6058; E-mail: joanne@chanlab.org

Submission: 26 August, 2020

Accepted: 28 September, 2020

Published: 30 September, 2020

Copyright: (๑) 2020 Richert-Jones J, et al. This is an open access article distributed under the Creative Commons Attribution License, which permits unrestricted use, distribution, and reproduction in any medium, provided the original work is properly cited.

\section{Materials and Methods}

\section{Fibroblast assays}

Keloid fibroblasts (CRL1762) and race-matched normal fibroblasts (CRL2439) were purchased from the American Type Culture Collection (ATCC; 217 Perry Parkway Gaithersburg, MD 20877, USA) and cultured in DMEM (Gibco cat. no. 1995-065; Life Technologies Limited 3 Fountain Drive Paisley, PA49RF, UK) containing 10\% FBS, 1:500 amphotericin B, and 1:100 penicillin/ streptomycin (Gibco cat. no. 26140-079, 15290-026, 15140-122; Life Technologies Corporation 3175 Staley Road Grand Island, NY 14072, USA) as described [9]. Cell viability was determined by hemocytometry and Trypan Blue dye exclusion. Equal cell numbers were plated at day 0 , then treated under 8 distinct conditions. Chemical inhibitors used were: 2-deoxyglucose (2-DG; SigmaAldrich cat. no. D6134; 3050 Spruce Street St. Louis, MO 63103, USA) at $10 \mathrm{mM}$ for 2-16 h, or $40 \mu \mathrm{M} \mathrm{KC2F7} \mathrm{for} 16 \mathrm{~h}$, NVP-AEW541, MLN1117 and BEZ235 (Selleck Chemicals cat. no. S7946; 14408; no. S1034, S8581, S1009; W Sylvanfield Drive Houston, TX 77014, USA). For immunofluorescence, cells were fixed, then permeabilized and stained with an anti-SMA or anti-fibronectin antibody (SigmaAldrich/MilliporeSigma, cat. No. A5228, MAB1926; 400 Summit Drive, Burlington, MA 1803, USA), Alexa Fluor 594 phalloidin, Alexa Fluor 488 phalloidin, Hoechst-33342 (Life Technologies cat. no. A12349, A12381, H1399; 29851 Willow Creek Road Eugene, OR 97402, USA), and/or DAPI (Molecular Probes, Inc. cat. no. 62248; 3747 N Meridian Road Rockford, IL 61101, USA). For live imaging, propidium iodide (BioSure, Inc. cat. no. 1032; 1050 Whispering Pines Lane, STE F, Grass Valley, CA 95945, USA) is used to stain dying cells and Hoechst-33342 is used to stain nuclei. Western blot analysis was performed as previously described [9]. Twenty micrograms of cell lysates per lane was used in standard western analysis. Blots were incubated with the indicated primary antibodies at $4{ }^{\circ} \mathrm{C}$, overnight, the appropriate secondary horseradish peroxidase (HRP)conjugated IgG (Life Technologies) used at 1:4000 in TBST for $1 \mathrm{~h}$ at RT. Immunoreactive protein bands were detected using Western 


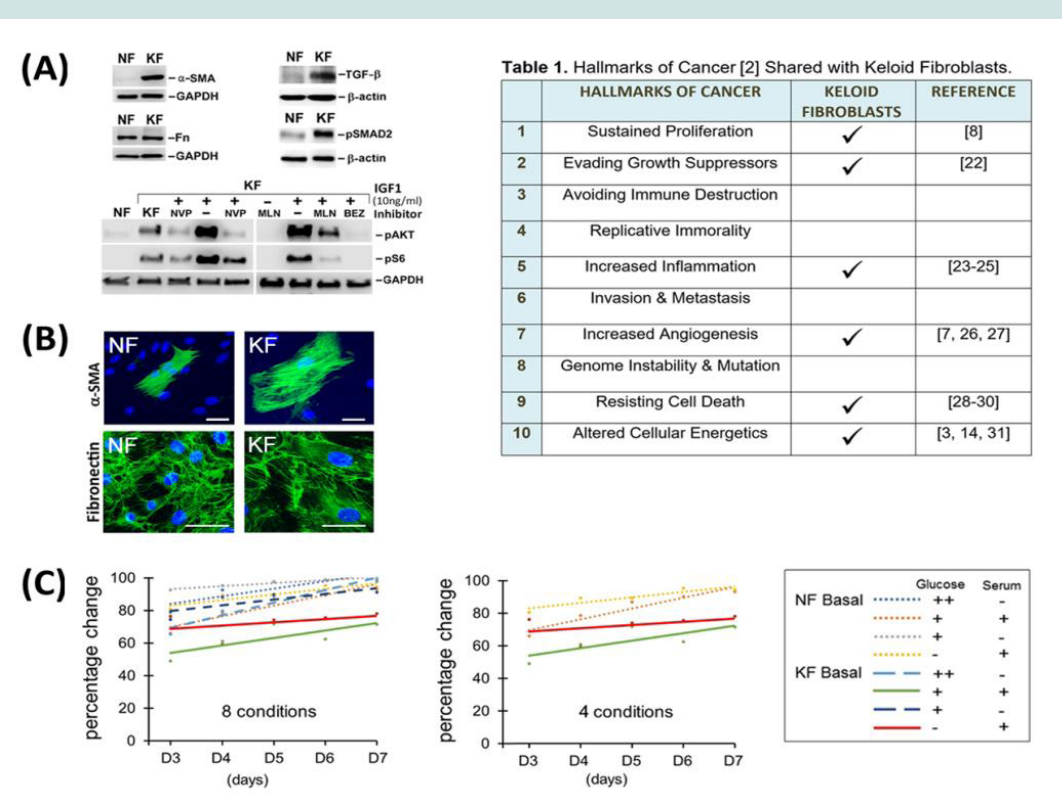

Figure 1: Keloid fibroblast characteristics and viability. (A) Western analysis of Keloid Fibroblasts (KF) protein expression and signaling pathway activation compared with control, Normal Fibroblasts (NF). Elevated expression of $\alpha$-SMA, fibronectin, TGF $\beta$ and pSMAD2 in unstimulated KFs. Analysis of IGF1 (10 ng/ml) signaling in KF and NF demonstrates activation of pAKT, pS6 at 30 min after stimulation. For KF, these kinases were activated at the basal state. Inhibitors (NVP, $1 \mu$ M NVPAEW541; MLN, 34M MLN1117; BEZ, 100nM BEZ235) were added 30 min before IGF1 stimulation in lanes as indicated. Twenty microgram of cell lysate was loaded in each lane. GAPDH or $\beta$-actin were used to show equal loading. (B) Immunofluorescent staining of (keloid fibroblasts), using $\alpha$-SMA, fibronectin, or phalloidin as indicated; nuclei were stained with DAPI in blue. Scale bar, $100 \mu \mathrm{m}$. (C) Percentage change in viability plotted for conditions specified in the legend. Glucose (++, 4.5g/L; +, 1g/L) and/or serum (+, present; -, absent) changes from basal, complete media are indicated. These changes did not reach statistical significance $(p<0.05)$ using ANCOVA; 4 conditions showing the greatest difference are also indicated (middle panel).

blot luminol reagent (Bio-Rad cat. no. P10026378/ P10026379, 1000 Alfred Nobel Drive Hercules, CA, USA). Primary antibodies were: phospho-SMAD2 (pSMAD2) and phospho-S6 (pS6) (Cell Signaling Technology, Inc. cat. no. $18338 \mathrm{~T}$ and no. 4857S, respectively; 3 Trask Lane, Danvers, MA 01923, USA).

\section{Statistical analysis}

The results were expressed as the mean \pm Standard Deviation (SD). Statistical analyses were performed using the SPSS 22.0 software, one-way ANCOVA for group comparisons or a paired T-test for pairwise comparisons. A value of $\mathrm{p} \leq 0.05$ was considered statistically significant.

\section{Results and Discussion}

Keloids are often compared with cancers in terms of their chronic and persistent growth. Some of these features are preserved in keloid fibroblasts (KFs); therefore, with the absence of an animal model, fibroblasts derived from keloid scars provide an important cellbased model that retains key features of keloids. Of the 10 hallmarks of cancer, keloids share 6 of them [2] (Table 1). To validate some of these characteristics [1], we used western and immunofluorescent analyses to examine the expression of relevant proteins and activated pathways in keloid fibroblasts (KFs), but not in normal skin fibroblasts (NFs; Figure 1A). KFs typically express high levels of Smooth Muscle Actin (SMA), a recognized property of the transitional myofibroblast [10]. As reported by others [11], we also noted expression of TGF- $\beta$ and detection of phospho-SMAD2 (pSMAD2) indicating chronic pathway activation in unstimulated KFs. In addition, mTORC1 (mammalian or mechanistic target of rapamycin complex 1) activity is also elevated in KFs, as shown by phospho-S6 (pS6) detection (Figure 1A). To demonstrate specificity of these activated signals, we also used IGF1 stimulation, either alone or in the presence of highly selective chemical inhibitors to block the signaling pathway at several nodes: IGF1 receptor (using NVP-AEW541), PI3Ka (phosphoinositide 3-kinase-alpha, using the PI3Ka inhibitor MLN1117), or both PI3K and mTORC1 (using the dual inhibitor NVP-BEZ235 [12,13]). In addition, we also observed an enlargement in KF cell size, in accord with enhanced mTORC1 activity, shown by immunofluorescent staining of SMA [5] (Figure 1B). Although the expression of some proteins is highly elevated, others are not. For example, fibronectin expression is similar in both types of fibroblasts (Figure1A and 1B).

Previous studies have shown a preference for glycolytic metabolism in KFs, where chemical inhibitors against this pathway were used to evaluate their potential use in keloid therapy $[3,14]$. In a comprehensive study by Vincent et al. (2008); [3]) changes in lactate or ATP production were reduced when glycolysis was impaired by 3 structurally distinct inhibitors. In another study, Li et al. (2018; [14]), 2-Deoxyglucose (2-DG) was shown to reduce KF viability over 4 days. Although both studies suggested that chemical glycolysis blockade could be targeted in treating keloids, it is not known whether KFs can be selectively inhibited while allowing NFs to survive.

To examine this further, we investigated whether limiting glucose availability could reveal a difference in viability between NF and KF. Thus, we determined live cell numbers when these fibroblasts were grown in media with reduced glucose and/or serum, as compared with 


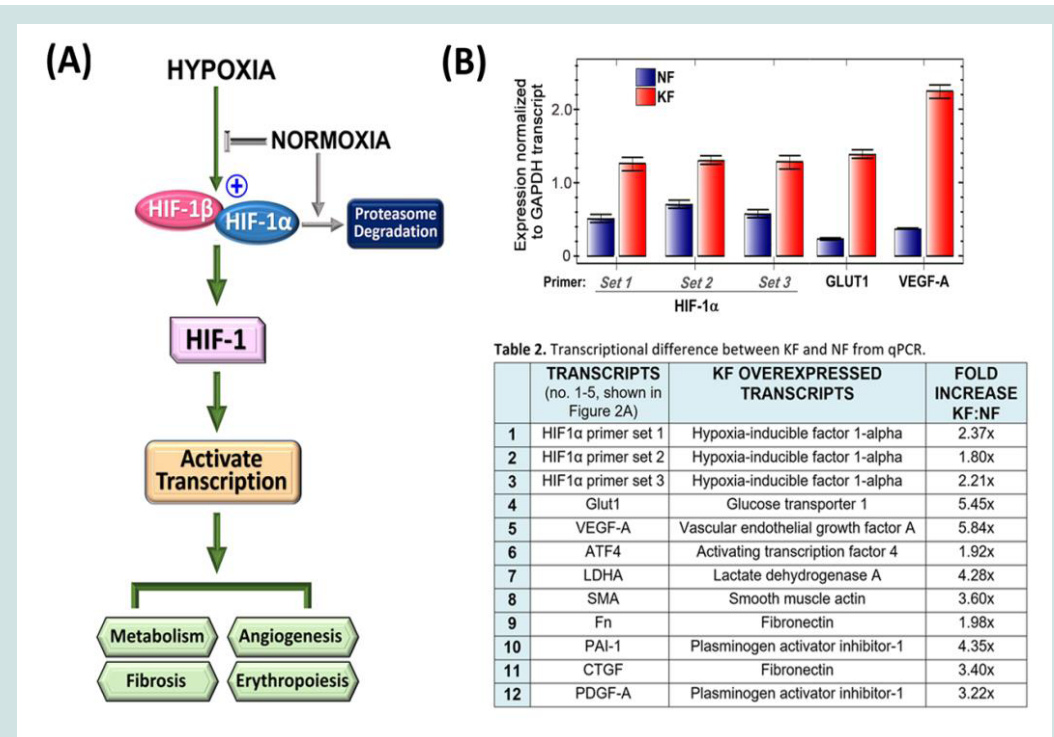

Figure 2: The HIF-1 pathway is activated in unstimulated keloid fibroblasts, affecting metabolism and angiogenesis. (A) The hypoxia pathway flowchart demonstrates activation of the HIF-1 transcription factor when the HIF-1a subunit is available to bind HIF-1b. Under normal oxygen levels, HIF-1a is synthesized as a protein, but is degraded before interaction with the HIF- $1 \mathrm{~b}$ subunit. (B) qPCR analysis of transcript levels for HIF-1a and HIF-1 regulated genes, GLUT1 and VEGF-A, in nstimulated NF or KF normalized to the expression of GAPDH. Three sets of HIF-1a primers were used to ensure accurate estimation.

complete media. For each type of fibroblast, at least 300,000 cells per flask were seeded in triplicate on day 0 . Cell numbers were counted from days 3-7 using hemocytometry (Figure 1C). To highlight glucose-specific effects, we presented a simplified graph to show that both NF and KF cells were affected at similar rates when glucose levels are reduced to $22 \%$ or $0 \%$. Thus, limiting glucose availability or antagonizing enzymes in the glycolysis pathway may not provide an effective means for keloid treatment. This observation was confirmed using an Analysis of Co-Variance (ANCOVA), to show that lowering glucose and/or serum levels did not yield statistical differences between cells ( $p>0.05$; Figure 1C).

We also examined whether preclinical drugs antagonizing overactivated pathways in KFs could destroy this abnormal cell type (Figure 1, data not shown). These include 2- DG to inhibit glycolysis, MLN-1117 to inhibit PI3K, BEZ235 to antagonize both PI3K and mTORC1, as well as KC7F2 (inhibiting HIF-1 $\alpha$; [15]). We reasoned that antagonizing the HIF-1 signaling pathway would limit the expression of genes for both glycolysis and fibrosis, which would be beneficial in keloid treatment (Figure 2A). HIF-1 targeting has also been considered for the treatment of muscle and organ fibrosis [16-18]. However, KFs are cultured under normoxic conditions; therefore, an abnormal activation of the hypoxia signaling pathway needs to be present in order for inhibitors to work. Elevated HIF-1 activity has been reported in KFs. In the Vincent et al. (2008) study, overactivation of hypoxia signaling in KFs was confirmed as these cells were still able to generate ATP in the presence of 2 hypoxia mimics: desferrioxamine and cobalt chloride [3]. We examined HIF$1 \alpha$ transcript levels using qPCR to find an $\sim 2$-fold increase. Using 3 distinct sets of primers, we confirmed HIF- 1 a overexpression in KFs at approximately $\sim 2$-fold, with a range of 1.6 to 2.3 -fold, compared with NFs. Although HIF-1 $\alpha$ mRNA was not highly overexpressed, HIF-1 regulated genes, GLUT1 (glucose transporter-1) and VEGF-A (vascular endothelial growth factor-A) transcripts, were increased in excess of 5-fold (Figure 2 and Table 2; see supplemental information). Thus, using protein or transcript detection, we have demonstrated increased TGF- $\beta$, GLUT1 and VEGF levels in the basal, unstimulated state in KFs. Since overactivation of these pathways can impact fibrosis, metabolism and angiogenesis, antagonizing HIF-1 function could affect several pathways that are abnormally activated in KFs (Figure 2A, 2B).

To determine whether a combined blockade of HIF-1a and glycolysis might be effective in eradicating KFs, we used a HIF-1a inhibitor, KC7F2 [15], either alone or in combination with 2-DG, on KF viability. Within $24 \mathrm{~h}$ of treatment, KFs were eliminated. Therefore, we refined conditions to obtain $\sim 50 \%$ reduction in KF viability (Figure 3A-3C). When 2-DG was present alone, cell viability was only reduced to $80 \%$. In the combined treatment, KF cell numbers remained similar to $\sim 50 \%$, as observed with KC7F2 treatment alone, suggesting that 2-DG did not contribute to enhancing KF elimination (Figure 3C). Furthermore, vital staining showed that increased cell death, indicated by propidium iodide, occurred in the presence of the KC7F2 HIF-1 $\alpha$ inhibitor (Figure 3A). In addition, immunofluorescent analysis demonstrated severe alterations in cell morphology in cells treated with the HIF-1 $\alpha$ inhibitor but not with 2-DG (Figure 3B). Thus, in contrast to previous studies [3,14], our data demonstrated that blocking glycolysis does not lead to the elimination of the abnormal KF cells, an important goal for any keloid therapy that aims to reduce recurrence and promote healing.

HIF-1 is a master regulator that controls essential processes including metabolism, fibrosis, angiogenesis and erythropoiesis (Figures 2A and 3D). Small molecule inhibitors that alter the functional levels of the HIF-1 $\alpha$ subunit have been in preclinical and clinical development for cancer treatment and anemia $[19,20]$. Currently, one of the HIF prolyl hydroxylase inhibitors, FG-4592 (Roxadustat, clinically approved in China in December 2018), has 


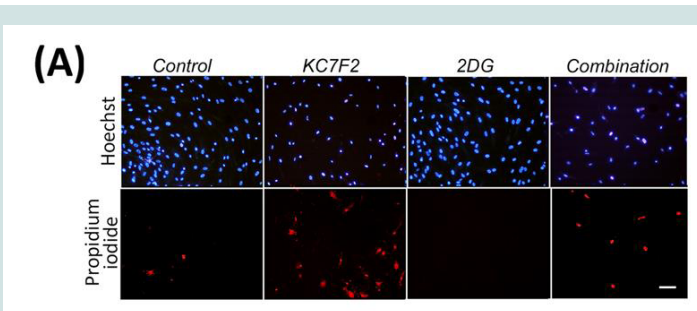

(B)

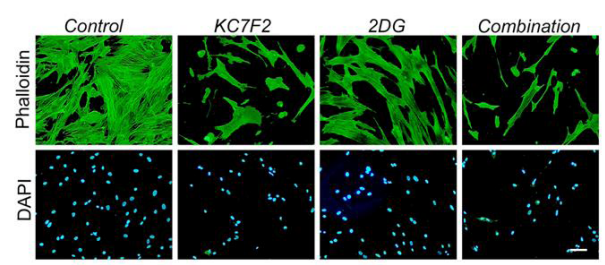

(C)

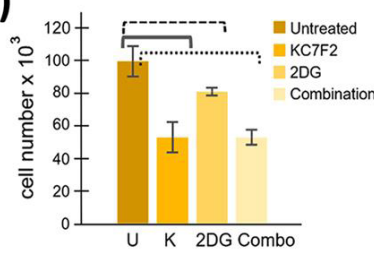

(D)

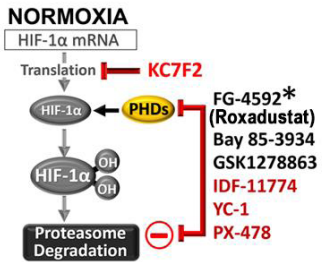

Figure 3: Keloid fibroblasts are more efficiently targeted by HIF1 $\alpha$ inhibition. (A, B) KFs were untreated, or treated with $40 \mu \mathrm{M} \mathrm{KC7F2,} \mathrm{and/or} 10 \mathrm{mM} 2 \mathrm{DG}$. (A) Vital staining of KFs using Hoescht 33342 to label nuclei (top) and propidium iodide to detect dying cells (bottom). (B) The same treatment was performed again where cells were fixed and stained with phalloidin (top) and DAPI (bottom). (C) Nuclei counts are presented. A pairwise T-test demonstrated statistical significance between untreated control KFs $(U)$ and all other conditions ( $p \leq 0.05)$; against KC7F2 alone $(K$, at $p=0.05)$; against 2-DG ( $p=0.04)$; or against the combination $(p=0.002)$. (D) The transcriptional activity of HIF-1 can be modified by chemical compounds that interfere with the expression and processing of HIF-1 $\mathrm{a}$. Prolyl hydroxylation of HIF-1a targets it for degradation. Prolyl Hydroxylase Domain (PHD) proteins can be inhibited by small molecule inhibitors such as FG-4592 ( ${ }^{*}$ Roxadustat, approved in China for anemia treatment), BAY 85-3934 or GSK1278863. Additional inhibitors such as KC7F2, YC-1, IDF-11774, and PX-478 reduce HIF-1a protein levels.

been successful in clinical phase II and III trials for treating anemia in patients with chronic kidney disease [21]. As more HIF-1a inhibitors become clinically approved, their additional use for keloid disease could be accelerated based on clinical safety data. For keloid disease in particular, these inhibitors can be locally administered through an intralesional injection. Thus, direct targeting of KFs may be achieved without inducing an additional skin injury.

\section{Conclusion}

Our study provides a novel drug target and treatment strategy for patients with keloid disease. This method may be extended to other skin disorders such as localized scleroderma, hidradenitis suppurativa, nephrogenic fibrosing dermopathy, and eosinophilic fasciitis, where localized fibrotic lesions persist.

\section{Data Availability}

Data presented in this manuscript is available to the research community without restriction at the time of online publication.

\section{References}

1. Trace AP, Enos CW, Mantel A, Harvey VM (2016) Keloids and Hypertrophic Scars: A Spectrum of Clinical Challenges. Am J Clin Dermatol 17: 201-223.

2. Hanahan D, Weinberg RA (2011) Hallmarks of cancer: the next generation. Cell 144: 646-674.

3. Vincent AS, Mukhopadhyay M, Lim HY, Barry Halliwell B, Wong KP, et al. (2008) Human skin keloid fibroblasts display bioenergetics of cancer cells. J Invest Dermatol 128: 702-709.

4. Syed F, Sanganee HJ, Singh S, Bahl A, Bayat A (2013) Potent dual inhibitors of TORC1 and TORC2 complexes (KU- 0063794 and KU-0068650) demonstrate in vitro and ex vivo anti-keloid scar activity. J Invest Dermatol 133: $1340-1350$

5. Syed F, Sherris D, Paus R,Varmeh S, Singh S, et al. (2012) Keloid disease can be inhibited by antagonizing excessive mTOR signaling with a novel dual
TORC $_{1 / 2}$ inhibitor. Am J Pathol 181: 1642- 1658.

6. Zhang Q, Wu Y, Ann DK, Messadi DV, Tuan TL, et al. (2003) Mechanisms of hypoxic regulation of plasminogen activator inhibitor-1 gene expression in keloid fibroblasts. J Invest Dermatol 121: 1005-1112.

7. Wu Y, Zhang Q, Ann DK, Akhondzadeh A, Duong HS, et al. (2004) Increased vascular endothelial growth factor may account for elevated level of plasminogen activator inhibitor-1 via activating $\mathrm{ERK}_{1 / 2}$ in keloid fibroblasts. Am J Physiol Cell Physiol 286: 905-912.

8. Russell SB, Trupin KM, Rodríguez-Eaton S, Russell JD, Trupinet JS, et al (1988) Reduced growth-factor requirement of keloid-derived fibroblasts may account for tumor growth. Proc Natl Acad Sci USA 85: 587-591.

9. Mantel A, Newsome A, Thekkudan T, Frazier R, Katdare M et al. (2016) The role of aldo-keto reductase $1 C_{3}\left(A_{K R} 1 C_{3}\right)$-mediated prostaglandin $D_{2}\left(P_{G D}\right)$ metabolism in keloids. Exp Dermatol 25: 38-43

10. Limandjaja GC, Belien JM, Scheper RJ, Niessen FB, Gibbset S, et al. (2019) Hypertrophic and keloid scars fail to progress from the $C_{34}{ }^{-1}(\alpha-S M A)^{+}$ immature scar phenotype and show gradient differences in alpha-SMA and P16 expression*. Br J Dermatol 182: 974-986.

11. Zhao MJ, Chen SY, Qu XY, Abdul-Fattah B, Lai T (2018) Increased cthrc1 activates normal fibroblasts and suppresses keloid fibroblasts by inhibiting TGF-beta/Smad signal pathway and modulating YAP subcellular location. Curr Med Sci 38: 894-902.

12. Garcia-Echeverria C, Pearson MA, Marti A, Meyer T, Mestan J, et al. (2004) In vivo antitumor activity of NVP-AEW541-A novel, potent, and selective inhibitor of the IGF-IR kinase. Cancer Cell 5: 231- 239.

13. Dienstmann R, Rodon J, Erra V, Tabernero J (2014) Picking the point of inhibition: a comparative review of PI3K/AKT/mTOR pathway inhibitors. Mol Cancer Ther 13: 1021-1031.

14. Li Q, Qin Z, Nie F, Bi H, Zhao R, et al. (2018) Metabolic reprogramming in keloid fibroblasts: Aerobic glycolysis and a novel therapeutic strategy. Biochem Biophys Res Commun 496: 641-647

15. Narita T, Yin S, Gelin CF, Moreno CS, Yepes M, et al. (2009) Identification of a novel small molecule HIF-1alpha translation inhibitor. Clin Cancer Res 15: 6128-6136. 
Citation: Richert-Jones J, Mantel A, Ricks-Santi L, Harvey VM, Chan J. Targeting Keloid Fibroblasts by Inhibition of Hypoxia Signaling. J Clin Investigat Dermatol. 2020;8(2): 5

\section{ISSN: 2373-1044}

16. Haase VH (2009) Pathophysiological Consequences of HIF Activation: HIF as a modulator of fibrosis. Ann N Y Acad Sci 1177: 57-65

17. Valle-Tenney R, Rebolledo D, Acuña MJ, Brandan E, et al. (2020) HIF hypoxia signaling in skeletal muscle physiology and fibrosis. J Cell Commun Signal 14: 147-158

18. Vanderstraeten J, Derradji H, Sonveaux P, Colin IM, Many MC, et al. (315) Acute iodine deficiency induces a transient VEGF- dependent microvascular response in mammary glands involving HIF-1, ROS, and mTOR. Am J Physiol Cell Physiol 315: 544-557.

19. Falla J, Rini BI (2019) HIF inhibitors: status of current clinical development. Curr Oncol Rep 21: 6.

20. Kular D, Macdougall IC (2019) HIF stabilizers in the management of renal anemia: from bench to bedside to pediatrics. Pediatr Nephrol 34: 365-378.

21. Chen N, Hao C, Liu BC, Lin H, Wanget C, et al. (2019) Roxadustat for Anemia in Patients with Kidney Disease Not Receiving Dialysis. N Engl J Med 381: 1001-1010.

22. Myles ME, Russell JD, Trupin JS, Smith JC, Russell SB, et al. (1992) Keloid fibroblasts are refractory to inhibition of DNA synthesis by phorbol esters. Altered response is accompanied by reduced sensitivity to prostaglandin E2 and altered down-regulation of phorbol ester binding sites. J Biol Chem 267 9014-9020.

23. Shaker SA, Ayuob NN, Hajrah NH (2011) Cell talk: a phenomenon observed in the keloid scar by immunohistochemical study. Appl Immuno Histochem Mol Morphol 19: 153-159

24. Murao N, Seino KI, Hayashi T, Ikeda M, Funayama E, et al. (2014) Treg- enriched CD4+ T cells attenuate collagen synthesis in keloid fibroblasts. Exp Dermatol 23: 266-271.

25. Gui L, Niu F, Bing Yu, Lauda N, Liu J, et al. (2018) Macrophages in keloid are potent at promoting the differentiation and function of regulatory $T$ cells. Exp Cell Res 362: 472-476.

26. Steinbrech DS, Mehrara BJ, Chau D, Rowe NM, Chin G, et al. (1999) Hypoxia upregulates VEGF production in keloid fibroblasts. Ann Plast Surg 42: 514519.

27. Fujiwara M, Muragaki Y, Ooshima A (2005) Upregulation of transforming growth factor-beta1 and vascular endothelial growth factor in cultured keloid fibroblasts: relevance to angiogenic activity. Arch Dermatol Res 297: 161169.

28. Appleton I., Brown NJ, Willoughby DA (1996) Apoptosis, necrosis, and proliferation: possible implications in the etiology of keloids. Am J Pathol 149: 1441-1447.

29. Sayah DN, Soo C, Shaw WW, Watson J, Messadi D, et al. (1999) Downregulation of apoptosis-related genes in keloid tissues. J Surg Res 87: 209-216.

30. Chodon T, Sugihara T, Igawa HH, Funayama E, Furukawa H (2000) Keloid-derived fibroblasts are refractory to Fas-mediated apoptosis and neutralization of autocrine transforming growth factor-beta1 can abrogate this resistance. Am J Pathol 157: 1661-1669.

31. Ishihara $H$, Yoshimoto $H$, Fujioka M, Murakami R, Hirano A, et al. (2000) Keloid fibroblasts resist ceramide-induced apoptosis by overexpression of insulin-like growth factor I receptor. J Invest Dermatol 115: 1065-1071. 